
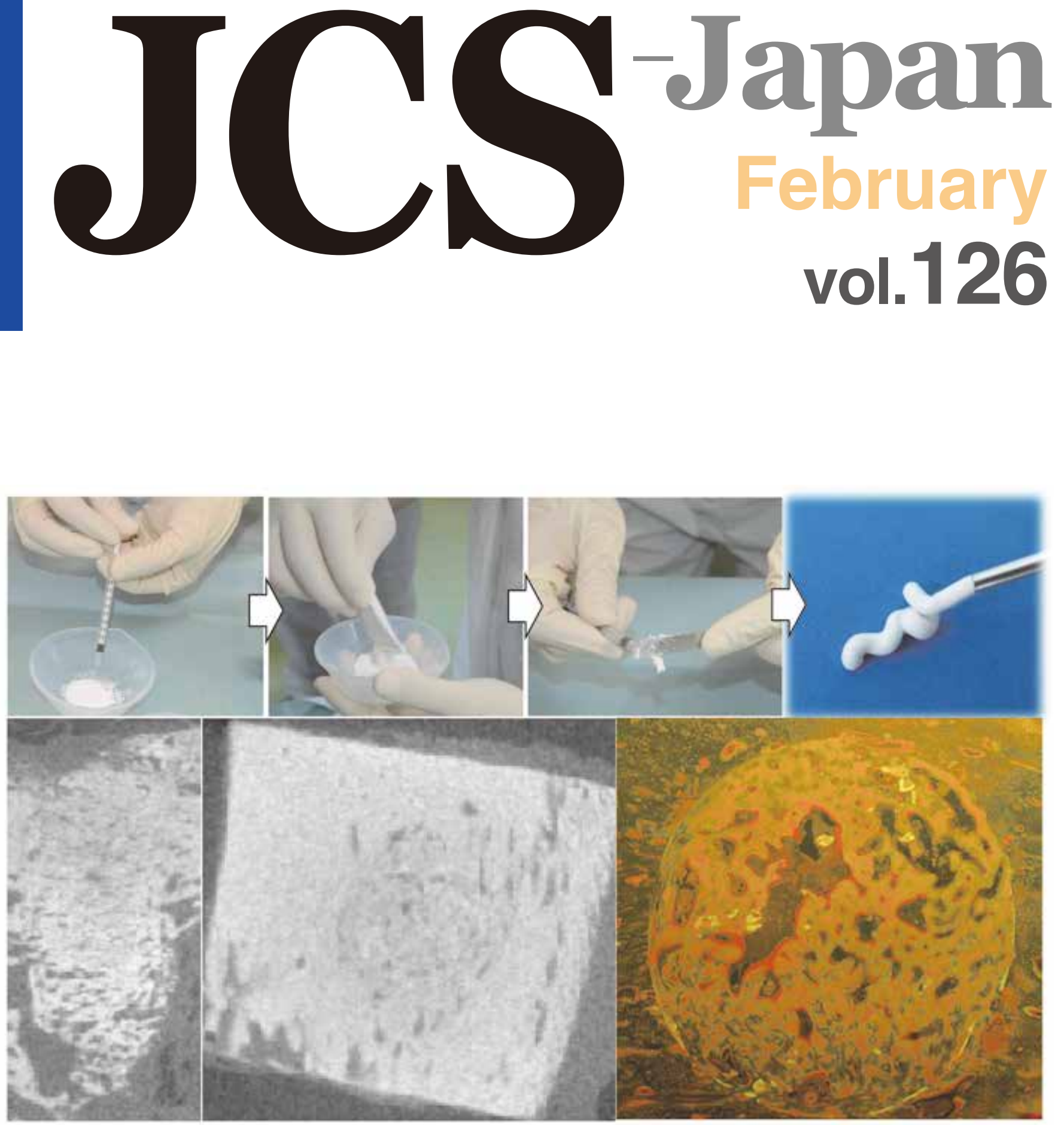

Journal of the Ceramic Society of Japan 


\title{
Bioresorbability of chelate-setting calcium-phosphate cement hybridized with gelatin particles using a porcine tibial defect model
}

\author{
Keishi KIMINAMI ${ }^{1,2}$, Kohei NAGATA ${ }^{1}$, Toshiisa KONISHI ${ }^{3}$, Minori MIZUMOTO ${ }^{4}$, \\ Michiyo HONDA ${ }^{1}$, Kazuaki NAKANO ${ }^{5}$, Masaki NAGAYA ${ }^{6}$, Hidetoshi ARIMURA ${ }^{2}$, \\ Hiroshi NAGASHIMA ${ }^{5,6}$ and Mamoru AIZAWA ${ }^{1,6, \dagger}$ \\ ${ }^{1}$ Department of Applied Chemistry, School of Science and Technology, Meiji University, \\ 1-1-1 Higashimita, Tama-ku, Kawasaki 214-8571, Japan \\ ${ }^{2}$ GUNZE LIMITED, 1 Zeze, Aono-cho, Ayabe, Kyoto 623-8512, Japan \\ ${ }^{3}$ Department of Medical Bioengineering, Graduate School of Natural Science and Technology, Okayama University, \\ 3-1-1 Tsushima-naka, Kita-ku, Okayama 700-8530, Japan \\ ${ }^{4}$ Kanagawa Academy of Science and Technology, 3-2-1 Sakado, Takatsu-ku, Kawasaki 213-0012, Japan (former affiliation) \\ ${ }^{5}$ Department of Life Sciences, School of Agriculture, Meiji University, 1-1-1 Higashimita, Tama-ku, Kawasaki 214-8571, Japan \\ ${ }^{6}$ Meiji University International Institute for Bio-resource Research (MUIBRR), \\ 1-1-1 Higashimita, Tama-ku, Kawasaki 214-8571, Japan
}

\begin{abstract}
Calcium-phosphate cements (CPCs) are widely used to reconstruct and augment bones. To enhance the clinical usefulness of these cements, researchers have put great effort into improving their material properties and bioresorbability. To create a novel bioresorbable CPC, we successfully incorporated gelatin particles into a chelate-setting CPC, whose powder component consisted of hydroxyapatite surface-modified with inositol hexaphosphate and $\alpha$-tricalcium phosphate. We expected that interconnected macropores could be formed inside the cement specimen through the degradation of the gelatin particles, resulting in cellular infiltration, specimen bioresorption, and subsequent new bone formation. To verify this hypothesis, we evaluated the bioresorbability and bone-forming ability of a gelatin-hybridized CPC implanted for eight weeks into porcine tibial defects. We also assessed the effects on the bioresorbability of polysaccharides (chitosan and chondroitin 6-sulfate) included in the liquid component of the CPC. Micro-CT observations and histological evaluations revealed that the use of chondroitin 6-sulfate could lead to enhancement of the bioresorbability and boneforming ability. Of special note, the resorption rate reached nearly $85 \%$, and new bone was observed at the resorbed sites inside the specimen. We conclude that gelatin-hybridized chelate-setting CPC containing chondroitin 6-sulfate is a promising bone substitute for non-load-bearing applications.

(02018 The Ceramic Society of Japan. All rights reserved.
\end{abstract}

Key-words: Calcium-phosphate cement, Chelate-setting cement, Inositol phosphate, Gelatin particles, Bioresorbability, Porcine tibial defect

\section{Introduction}

Because calcium-phosphate cements (CPCs) have good injectability, biocompatibility, and osteoconductivity, they are promising biomaterials for use in minimally-invasive bone reconstruction and augmentation techniques. CPCs have certain drawbacks, however: they are easily washed out upon early contact with body fluid, and their bioresorbability is low because of the formation of hydroxyapatite (HAp) as a final product. ${ }^{1) 2}$ ) These challenges must be overcome to enhance the clinical usefulness of CPCs.

\footnotetext{
Corresponding author: M. Aizawa; E-mail: mamorua@ meiji.ac.jp
}

To address washout problems, we previously developed a chelate-setting CPC, whose powder component was a mixture of 1) an HAp powder surface-modified with inositol hexaphosphate (IP6) and 2) an $\alpha$-tricalcium phosphate $(\alpha$-TCP) powder with a mixing ratio of $20 / 80$ $[\mathrm{g} / \mathrm{g}] .{ }^{3)}$ This novel IP6-HAp $/ \alpha$-TCP cement was capable of setting, and exhibited washout resistance based on both chelate-bonding between IP6 and calcium ions and the entanglement of HAp crystals formed by $\alpha$-TCP hydrolysis. The liquid component contained polysaccharides (chitosan, chondroitin 6-sulfate, and sodium alginate) as cohesion promoters, meanwhile that made the CPC sufficiently injectable.

To enhance the bioresorbability of the chelate-setting CPC, moreover we recently successfully incorporated 
irregularly-shaped gelatin particles into the CPC. These particles have a broad size distribution and a median particle size of approximately $200 \mu \mathrm{m} .{ }^{4)}$ We anticipated that gradual degradation of the gelatin particles would enable sufficient formation of interconnected macropores inside the cement specimen. These macropores could promote cell infiltration, specimen bioresorption, and subsequent replacement by newly-formed bone.

Evaluation of the effects of the polysaccharides included in the liquid component on the material properties of the gelatin-hybridized CPC revealed that chondroitin 6-sulfate was the most suitable additive for obtaining a CPC with higher compressive strength $(\mathrm{CS}){ }^{4}{ }^{4}$ This result stemmed from interactions between gelatin particles and the negatively-charged sulfonyl groups of chondroitin 6-sulfate. We found that chitosan, by contrast, was able to provide the CPC with a relatively lower washout ratio, probably because of the formation of a polyelectrolyte complex between the positively-charged chitosan and the gelatin particles.

Prior to studying the bioresorbability of the gelatinhybridized CPC, we hypothesized that the use of chondroitin 6-sulfate rather than chitosan in the liquid component could contribute to enhancing the bioresorption rate of the CPC. This hypothesis was based on a previous report demonstrating that chondroitin sulfate was considered to increase the osteoconductivity of a CPC because its differently charged residues (i.e., carboxyl-, sulfate-, and amide-groups) immobilized growth factors and other cytokines on the CPC surface to stimulate cell activity. ${ }^{5)}$ In particular, we expected that osteoclasts could appear around the cement specimen at an early stage of implantation to facilitate gelatin degradation and subsequent cement resorption.

In this study, we evaluated the bioresorbability of a gelatin-hybridized CPC prepared with a liquid component containing chondroitin 6-sulfate or chitosan. The CPC was implanted for eight weeks into porcine tibial defects in order to optimize the composition of the CPC in terms of its bioresorbability and material properties.

\section{Material and methods}

\subsection{Preparation of $\alpha$-TCP powder}

Ten grams of commercially-available $\alpha$-TCP raw pow$\operatorname{der}(\alpha-T C P-A$; Taihei Chemical Industrial Co., Ltd., Osaka, Japan) were used as the starting material. The $\alpha$-TCP powder was ground for $60 \mathrm{~min}$ at a rotation rate of 300 rpm, using a planetary ball-mill (Pulverisette 6, Fritsch, Idar-oberstein, Germany) in a $\mathrm{ZrO}_{2}$ pot with $180 \mathrm{~g}$ of $\mathrm{ZrO}_{2}$ beads $2 \mathrm{~mm}$ in diameter under wet conditions $\left(40 \mathrm{~cm}^{3}\right.$ of distilled water). After ball-milling, the resulting slurry was filtered and freeze-dried for $24 \mathrm{~h}$ to yield "ball-milled $\alpha$ TCP powder."

\subsection{Preparation of HAp powder surface- modified with IP6}

A sodium inositol hexaphosphate (IP6-Na) solution was prepared at a concentration of $8000 \mathrm{ppm}$ using 50 mass \% phytic acid (Wako Pure Chemical Industries, Ltd., Osaka, Japan) and adjusted to a $\mathrm{pH}$ of 7.3 with an $\mathrm{NaOH}$ solution. Ten grams of commercially-available HAp powder (HAp100; Taihei Chemical Industrial Co., Ltd., Osaka, Japan) were simultaneously ground and surface-modified by ballmilling with $50 \mathrm{~cm}^{3}$ of the $8000 \mathrm{ppm}$ IP6-Na solution, using a planetary ball-mill for $60 \mathrm{~min}$ at a rotation rate of $300 \mathrm{rpm}$ in a $\mathrm{ZrO}_{2}$ pot with $180 \mathrm{~g}$ of $\mathrm{ZrO}_{2}$ beads $2 \mathrm{~mm}$ in diameter. After ball-milling, the resulting slurry was filtered and freeze-dried for $24 \mathrm{~h}$, to yield the "IP6-HAp powder."

\subsection{Preparation of thermally cross-linked gel- atin particles}

Gelatin particles extracted from porcine skin, alkalineprocessed, and ground into an average diameter of approximately $200 \mu \mathrm{m}$ (Nitta Gelatin Inc., Osaka, Japan) were heated at $140^{\circ} \mathrm{C}$ for $14 \mathrm{~h}$ under vacuum conditions. The thermally cross-linked gelatin particles were irradiated with $\gamma$-rays $(25 \mathrm{kGy})$.

\subsection{Preparation of liquid component for cement fabrication}

As shown in Table 1, three types of mixing solutions were prepared: "Chito-10", containing 10 mass $\%$ of chitosan (Daichitosan Coat GL, Dainichiseika Color \& Chemicals Mfg. Co., Ltd., Tokyo, Japan) and 2.5 mass \% of sodium dihydrogen phosphate, "Chondro-10" and "Chondro-15," containing 10 and 15 mass $\%$ of chondroitin 6sulfate sodium salt and 2.5 mass $\%$ of disodium hydrogen phosphate. All the solutions were adjusted to a $\mathrm{pH}$ of 7.0 with $\mathrm{NaOH}$ solution and sterilized with filtration. With the exception of chitosan, the other chemicals were purchased from Wako Pure Chemical Industries, Ltd., Osaka, Japan.

\subsection{Evaluation of material properties of cement pastes and set specimens}

The IP6-HAp powder was premixed with the ballmilled $\alpha$-TCP powder at a mixing ratio of $20 / 80[\mathrm{~g} / \mathrm{g}]$ for $5 \mathrm{~min}$, using a Super Micro V-Shape Mixer (MC, Tsutsui Scientific Instruments Co., Ltd., Tokyo, Japan). The premixed powder was mixed further with the prepared gelatin particles for an additional $5 \mathrm{~min}$ at a ratio of $100 / 0$

Table 1. Compositions of liquid components used to prepare cement pastes

\begin{tabular}{|c|c|c|c|}
\hline Abbreviation & Polysaccharide & Additive & $\mathrm{pH}$ \\
\hline Chito-10 & 10 mass \% of Chitosan & 2.5 mass $\%$ of Sodium dihydrogen phosphate & 7.0 \\
\hline Chondro-10 & 10 mass $\%$ of Chondroitin 6 -sulfate sodium salt & 2.5 mass $\%$ of Disodium hydrogen phosphate & 7.0 \\
\hline Chondro-15 & 15 mass $\%$ of Chondroitin 6-sulfate sodium salt & 2.5 mass $\%$ of Disodium hydrogen phosphate & 7.0 \\
\hline
\end{tabular}


Table 2. Compositions of cement pastes

\begin{tabular}{cccc}
\hline Abbreviation & $\begin{array}{c}\text { Gelatin particles } \\
{[\mathrm{mass} \%]}\end{array}$ & Mixing solution & $\begin{array}{c}\mathrm{P} / \mathrm{L} \text { ratio } \\
{\left[\mathrm{g} / \mathrm{cm}^{3}\right]}\end{array}$ \\
\hline Chito-10(G0) & 0 & Chito-10 & $1 / 0.5$ \\
Chito-10(G10) & 10 & Chito-10 & $1 / 0.7$ \\
Chondro-10(G0) & 0 & Chondro-10 & $1 / 0.5$ \\
Chondro-10(G10) & 10 & Chondro-10 & $1 / 0.7$ \\
Chondro-15(G0) & 0 & Chondro-15 & $1 / 0.5$ \\
Chondro-15(G10) & 10 & Chondro-15 & $1 / 0.7$ \\
\hline
\end{tabular}

or $90 / 10[\mathrm{~g} / \mathrm{g}]$. The cement pastes were prepared by mixing the obtained powder with the mixing solutions listed in Table 1 at the designated powder/liquid $(\mathrm{P} / \mathrm{L})$ ratios $\left[\mathrm{g} / \mathrm{cm}^{3}\right]$, as presented in Table 2 .

The initial setting time (IST) was measured using a light Gillmore needle (113.4 g) in accordance with JIS T $0330-4$. The prepared cement pastes were packed in plastic molds ( $8 \mathrm{~mm}$ in diameter and $2 \mathrm{~mm}$ in height), and the IST was measured at the desired times until the cement pastes were set. Three cement specimens were tested to obtain the average value with the standard deviation.

For the CS test, the set cement pastes were packed in cylindrical Teflon ${ }^{\circledR}$ molds $(6 \mathrm{~mm}$ in diameter and $12 \mathrm{~mm}$ in height), maintained at $37^{\circ} \mathrm{C}$ and $100 \%$ humidity for $1 \mathrm{~h}$, and then kept in distilled water at $37^{\circ} \mathrm{C}$ for $24 \mathrm{~h}$. CS tests were performed on the cement specimens using a universal testing machine (AG-5KNXplus, Shimadzu Co., Kyoto, Japan) in accordance with JIS T 0330-4. The crosshead speed was $0.5 \mathrm{~mm} / \mathrm{min}$, and a load cell of $5 \mathrm{kN}$ was used. Four cement specimens were tested to obtain the average value with the standard deviation.

Washout resistance tests were also conducted in accordance with JIS T 0330-4. At $5 \mathrm{~min}$ after mixing of the cement pastes, the pastes $\left(0.3 \mathrm{~cm}^{3}\right)$ were extruded from syringes onto wire gauze, after which the cement pastes on the wire gauze were immersed in saline $\left(30 \mathrm{~cm}^{3}\right)$. After $24 \mathrm{~h}$ of immersion at $37^{\circ} \mathrm{C}$, the cement specimens on the wire gauze and the washed-out specimens that had seeped through the gauze were removed and dried at $60^{\circ} \mathrm{C}$ for $24 \mathrm{~h}$. The washout ratios were calculated according to the following equation:

$$
\text { Washout ratio }(\%)=\frac{W_{\mathrm{B}}}{W_{\mathrm{A}}+W_{\mathrm{B}}} \times 100
$$

where $W_{\mathrm{A}}$ is the weight of the cement specimens on the wire gauze and $W_{\mathrm{B}}$ is the weight of the washed-out cement specimens underneath the wire gauze. Four cement specimens were tested to obtain the average value with the standard deviation.

\subsection{Evaluation of bioresorbability of cement specimens using a porcine tibial defect model}

The premixed IP6-HAp/ $\alpha$-TCP powder was sterilized with ethylene oxide gas. Immediately prior to injection into the porcine tibial defects, the prepared gelatin particles were added into the premixed powder at a mixing ratio of $0 / 100$ or $10 / 90[\mathrm{~g} / \mathrm{g}]$. The powder was mixed for a further
1.5 min with the Chito-10 and Chondro- 15 mixing solutions at the designated $\mathrm{P} / \mathrm{L}$ ratios $\left[\mathrm{g} / \mathrm{cm}^{3}\right]$ presented in Table 2. The obtained cement pastes $\left(0.15 \mathrm{~cm}^{3}\right)$ were packed into plastic syringes for the injection.

We used two female transgenic-cloned pigs to evaluate the bioresorbability of the cement pastes. Chito-10(G0) and Chito-10(G10) were implanted into Pig 1, weighing $96 \mathrm{~kg}$. Chondro-15(G0) and Chondro-15(G10) were implanted into Pig 2, weighing $124 \mathrm{~kg}$. These two pigs carried a gene for a newly developed red fluorescent protein, humanized Kusabira-Orange (huKO), which was cloned from Fungia concinna coral stone. ${ }^{6}$ ) The pigs had access to a standard laboratory diet and water throughout the studies.

Surgical procedures were performed according to the Guidelines for Animal Care and Use Committee of Meiji University. The right tibias of the pigs were exposed, and cylindrical defects $(4.0 \mathrm{~mm}$ in diameter) were drilled into the epiphysis of the tibias. Three cement specimens of each composition were injected into the defects through syringes. After eight weeks of implantation, the pigs were euthanized, and their tibias were harvested and cut down in preparation for CT scans of the specimens using a micro-focus X-ray system (inspeXio SMT-90CT, Shimadzu Corporation, Kyoto, Japan). For histological observation, the freshly isolated tibias were fixed with $70 \%$ ethanol and subjected to Villanueva bone staining and observation under a light microscope. For histomorphometric analysis, an image-analysis system (WinROOF, Mitani Co., Tokyo, Japan) was used to quantify the areas of the residual and resorbed cement specimens based on manually traced contours. Image-analysis software was used to determine the cross-sectional areas and to express these areas as bioresorption rates.

\subsection{Statistical analysis}

Results are presented as the mean \pm standard deviation. A two-way ANOVA (analysis of variance) was applied.

\section{Results}

\subsection{Material properties of cement pastes and set specimens}

Prior to the evaluation of bioresorbability, the material properties of the non-hybridized and gelatin-hybridized IP6-HAp/ $\alpha$-TCP cements listed in Table 2 were examined. Figure 1(A) shows that the IST was increased by the gelatin hybridization; all the cement pastes set within $10 \mathrm{~min}$, however, probably satisfying the clinical requirements. Figure 1(B) demonstrates that regardless of the mixing solution used, gelatin hybridization significantly decreased the CS of the set cement specimens due to the weaker gelatin phase. The CS declined to approximately 4$5 \mathrm{MPa}$, which falls within the range for cancellous bones. ${ }^{7)}$ Chondro-15(G10) had a slightly higher CS than Chito10(G10), meanwhile, which was in good agreement with our previous work. ${ }^{4)}$ Figure $1(\mathrm{C})$ indicates that the washout ratio increased with gelatin hybridization, presumably because gelatin particle swelling allowed water to penetrate 
A

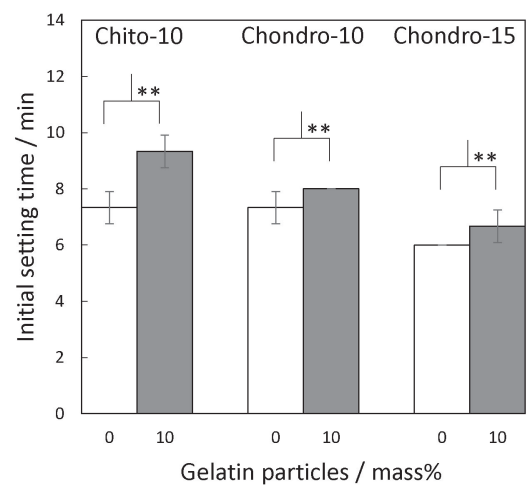

B

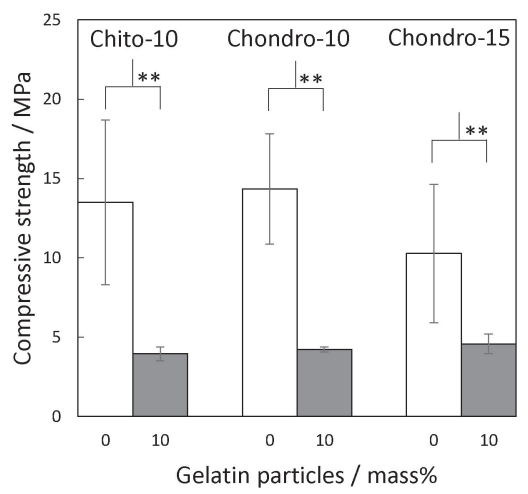

C

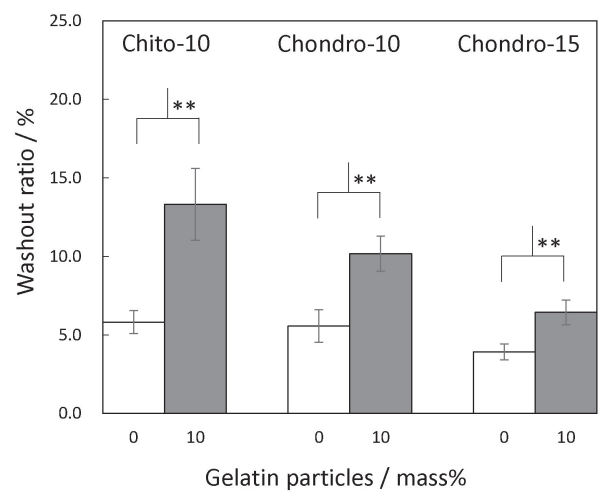

Fig. 1. (A) Initial setting time, (B) Compressive strength, (C) Washout ratio of the non-hybridized and 10 mass $\%$ gelatin-hybridized IP6-HAp $/ \alpha$-TCP cement specimens prepared with Chito-10, Chondro-10, and Chondro-15 mixing solutions at the $\mathrm{P} / \mathrm{L}$ ratios presented in Table 2. Significances: ${ }^{* *}=\mathrm{P}<0.01$.

A

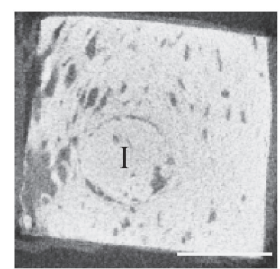

B

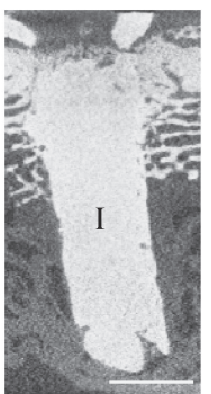

C

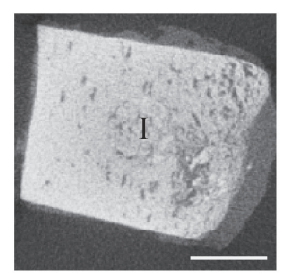

D

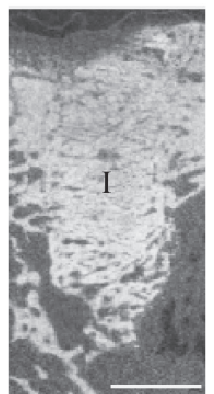

$\mathbf{E}$

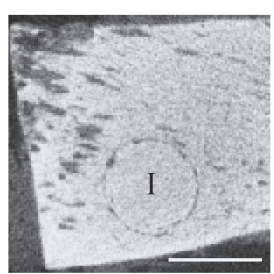

$\mathbf{F}$

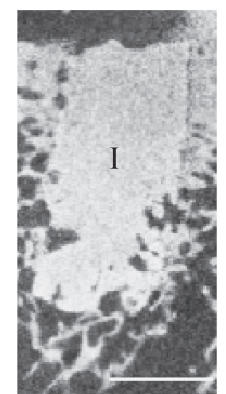

G
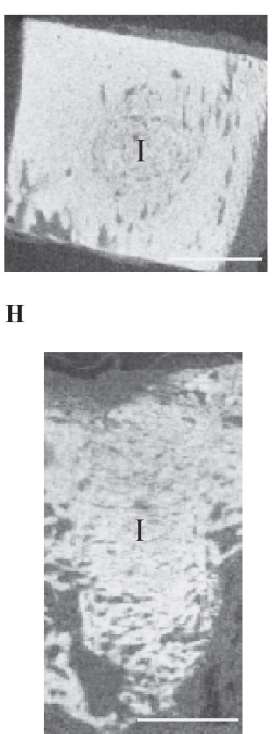

Fig. 2. Micro-focus X-ray CT images of (A, B) Chito-10(G0), (C, D) Chito-10(G10), (E, F) Chondro-15(G0), and $(\mathrm{G}, \mathrm{H})$ Chondro-15(G10) cement specimens implanted into porcine tibial defects for eight weeks. $I$ indicates the remaining cement specimens. (A), (C), (E), and (G) show horizontal views, and (B), (D), (F), and (H) show axial sectional views. Scale bars represent $4.0 \mathrm{~mm}$ in $(A),(C),(E)$, and $(\mathrm{G})$, and $3.0 \mathrm{~mm}$ in $(B),(D),(F)$, and $(H)$.

the cement paste. It was shown that Chondro-15(G10) exhibited a lower washout ratio than Chondro-10(G10), moreover, suggesting that the increased concentration of chondroitin sulfate contributed to improvement of its washout-resistance capabilities. Based on these results, we selected Chito-10(G0), Chito-10(G10), Chondro-15(G0), and Chondro-15(G10) as the cement compositions to be subjected to in vivo evaluation of bioresorbability.

\subsection{Effects of polysaccharides in mixing solutions on bioresorbability of cement pastes}

\subsubsection{Animal conditions}

Neither pig developed a bacterial infection or fracture during the course of the experiments.

\subsubsection{Micro-CT observations}

Micro-CT observations of the Chito- $10(\mathrm{G} 0)$ and Chondro-15(G0) cement specimens showed clear border lines between every specimen, with the respective surrounding tissues maintaining their shapes [Figs. 2(A), 2(B), 2(E), $2(\mathrm{~F})]$. It was anticipated that most of these specimens would remain intact without being resorbed. Fig. 2(F) also demonstrates that the surface profile of the Chondro15 (G0) cement specimen contacting the bone marrow was relatively rough, suggesting that the specimen was partially resorbed at this site.

Figures 2(C), 2(D) and 2(G), 2(H) show, by contrast, that the gelatin-hybridized Chito- $10(\mathrm{G} 10)$ and Chondro15(G10) cement specimens had relatively indistinguishable border lines with the respective host bones, probably 
A

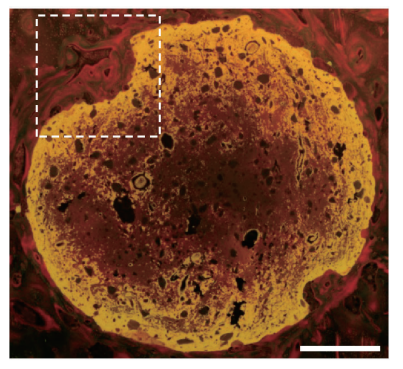

B

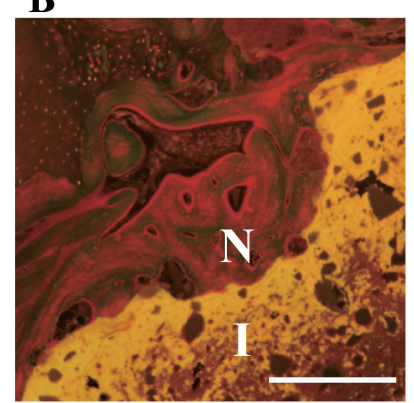

C

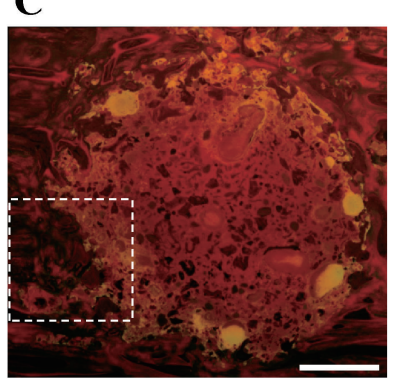

D

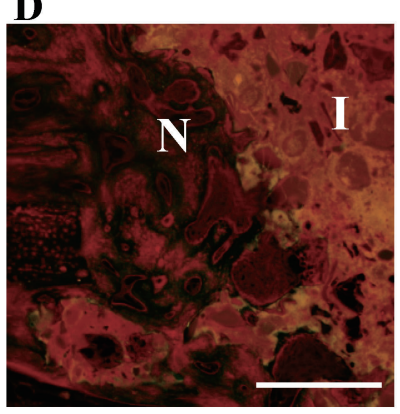

E

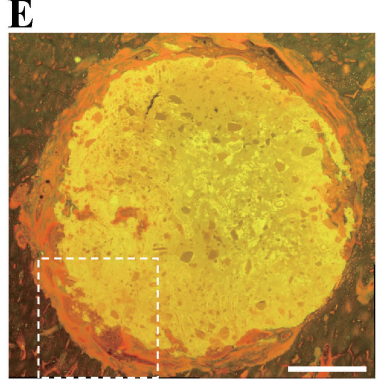

F

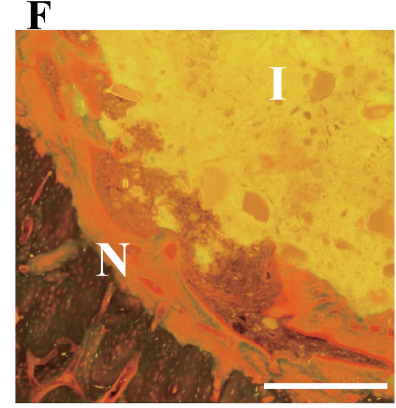

G

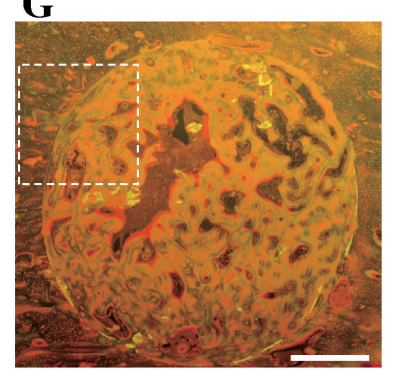

H

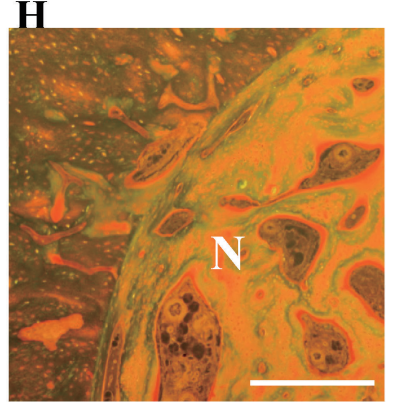

Fig. 3. Histological observations of (A, B) Chito-10(G0), (C, D) Chito-10(G10), (E, F) Chondro-15(G0), and $(\mathrm{G}, \mathrm{H})$ Chondro-15(G10) cement specimens implanted in porcine tibial defects for eight weeks (Villanueva bone stain). Low-magnification images $(A),(C),(E)$, and $(G)$ show the entire implanted cement specimens, and (B), (D), (F), and (H) show high-magnification images of the dotted square areas shown in (A), (C), (E), and (G), respectively. $I$ indicates remaining cement specimens. $N$ indicates newly-formed bone. Scale bars represent $1.0 \mathrm{~mm}$ in $(\mathrm{A}),(\mathrm{C}),(\mathrm{E})$, and $(\mathrm{G})$, and $500 \mu \mathrm{m}$ in $(\mathrm{B}),(\mathrm{D}),(\mathrm{F})$, and $(\mathrm{H})$.

because of peripheral resorption of the specimens promoted by gelatin degradation, and the subsequent infiltration of cells provided by the host tissues.

\subsubsection{Histological evaluations}

Histological observation of the Chito-10(G0) and Chito$10(\mathrm{G} 10)$ cement specimens showed that no fibrous tissue was apparent at the interface between the specimens and the surrounding bones [Figs. 3(A)-3(D)]. Histomorphometric analysis revealed, moreover, that only $3 \%$ of the Chito-10(G0) cement specimen was exclusively resorbed in the peripheral area [Figs. 3(A), 4]. When Chito-10(G10) cement specimens were implanted, by contrast, the average resorption rate increased by up to approximately $13 \%$, and hypo-calcified and calcified bone was found at the resorbed sites [Figs. 3(C), 3(D), 4]. The resorbed sites were limited to peripheral areas, however, and most of the specimen in the central area appeared to remain intact without being resorbed.

Figures 3(E) and 3(G) show that the Chondro-15(G0) and Chondro-15(G10) cement specimens also contacted the host bones directly with no interference from fibrous tissues. In addition, histomorphometric analysis demonstrated that the average resorption rate for the Chondro$15(\mathrm{G} 0)$ specimens reached $17 \%$ (Fig. 4), significantly higher than that for Chito-10(G0). Hypo-calcified and calcified bones were seen at resorbed peripheral sites [Figs. 3(E), 3(F)].

It was also revealed that gelatin hybridization of Chondro-15(G0) dramatically increased the average resorption rate to $85 \%$ (Fig. 4). Resorption sites were

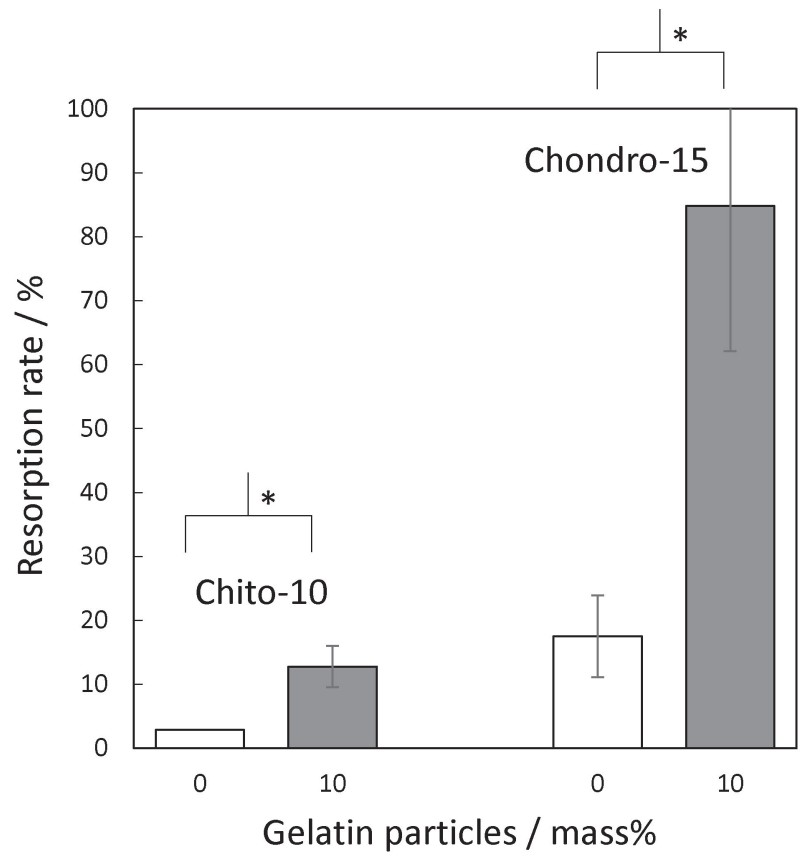

Fig. 4. Resorption rates for Chito-10(G0), Chito-10(G10), Chondro-15(G0), and Chondro-15(G10) cement specimens implanted in porcine tibial defects for 8 weeks. Significances: $*=\mathrm{P}<0.05$.

observed to be replaced by newly formed calcified bone extending from the bone surface area into the specimens [Figs. 4(G), 4(H)]. 
From these results, we can conclude that chondroitin 6-sulfate performed better than chitosan in terms of increased bioresorbability and bone-forming ability of the gelatin-hybridized cement specimens. Because of the good balance between the resorption of the specimens and the subsequent new bone formation, a gelatin-hybridized CPC fabricated with chondroitin 6-sulfate was expected to be a promising artificial bone graft with sufficient osteoconductivity, bioresorbability, and injectability.

\section{Discussion}

When bone defects caused by a congenital disease, trauma, or bone tumor removal surgery must be repaired, autografts are used as the gold standard for bone reconstruction and augmentation. Autografts have cetain drawbacks, however, such as invasiveness and limited availability. To establish alternatives to autografts, many types of artificial bone substitutes with different compositions, shapes, and material properties have been developed. Because of their injectability, CPCs are especially promising materials for minimally invasive repair of bone tissues. CPC bioresorption rates are low, however, because of the formation of HAp as the final product. For this reason, we expected the novel chelate-setting IP6-HAp/ $\alpha$-TCP cement that we developed recently to have low bioresorbability.

One effective strategy for enhancing CPC bioresorbability is to incorporate biodegradable solid-state polymers into the cement, causing the creation of macropores inside cement specimens as the polymers degrade. These newlyformed macropores are expected to facilitate the infiltration of cells and subsequent resorption of the cement specimens. While many types of polymers have been studied for this purpose, including poly(lactic acid-co-glycolic acid) (PLGA) ${ }^{8)-10)}$ and chitosan, ${ }^{11), 12)}$ gelatin seemed to us to be preferable, given our aim of enhancing the bioresorbability and bone-forming ability of our chelatesetting IP6-HAp/ $\alpha$-TCP cement. We based this hypothesis on previous reports demonstrating that gelatin tended to be sufficiently resorbed in vivo relative to PLGA, ${ }^{13)}$ and that initial cell adhesion and proliferation in CPCs could be promoted by the incorporation of gelatin. ${ }^{14), 15)}$ Habraken et al. successfully introduced gelatin microspheres with an average size of less than $10 \mu \mathrm{m}$ into a CPC. ${ }^{13), 16)}$ Kasuya et al., by contrast, used larger gelatin particles with a broad size distribution and an average particle size of approximately $200 \mu \mathrm{m}$ to enhance the bioresorbability of a CPC. These studies demonstrated that the gelatin-hybridized CPC was sufficiently resorbed and replaced by newly formed bone using a rabbit femoral defect model. ${ }^{17)}$ These events were attributed to the formation of interconnected macropores inside the cement specimens associated with gelatin degradation, which allowed for considerable cellular migration into the specimens.

Accordingly, in order to create a novel chelate-setting CPC with sufficient bioresorbability, we previously developed and evaluated the material properties of an IP6HAp $/ \alpha$-TCP cement hybridized with larger gelatin parti- cles. We expected to observe the formation of interconnected macropores and subsequent infiltration of cells when the gelatin-hybridized CPC was implanted in bone tissues.

In the present study, the bioresorbability of the newlycreated gelatin-hybridized CPC was evaluated by means of implantation into porcine tibial defects. We simultaneously examined the effects of polysaccharides (chitosan and chondroitin 6-sulfate) contained in the liquid component of the CPC on bioresorbability. Prior to the bioresorbability study, we conducted material property tests on the cement specimens.

The IST evaluation results showed that all of the cement pastes set within $10 \mathrm{~min}$; the hydrolysis of $\alpha$-TCP and chelate-bonding between IP6 and calcium ions were considered to contribute to the setting reaction. It was observed, moreover, that the 10 mass $\%$ gelatin hybridization did not significantly affect the IST of the cement specimens.

We demonstrated that gelatin hybridization considerably decreased the CS of the cement specimens, regardless of the type of liquid component, to approximately 4-5 MPa. These results indicate that the clinical application of gelatin-hybridized CPC should be limited to non-loadbearing sites.

In addition, gelatin hybridization was found to increase the washout ratio of the cement specimens. This was probably because of swelling of gelatin particles allowing water to penetrate the cement pastes. We consider, however, that all the gelatin hybridized cements retain enough washout-resistance capabilities, since no washout of the specimens was observed in the present in vivo study. Nonetheless, further evaluation using in vivo models in which the cement specimens are more severely exposed to blood and other body fluids is of great importance. The present washout-resistance tests also demonstrated that Chondro-15(G10) showed a lower washout ratio than Chondro-10(G10). This was probably because the viscosity or cohesiveness of the prepared cement paste was enhanced with the increased concentration of chondroitin sulfate acting as a cohesion promoter.

Micro-CT observations and histological evaluations of the cement specimens implanted into porcine tibial defects indicated that all the specimens contacted the surrounding host bones directly, demonstrating their biocompatibility and osteoconductivity. Micro-CT observations showed clear border lines, moreover, between the two nonhybridized cement specimens and their respective host bones. In addition, histological evaluations revealed that the majority of these specimens remained intact, without being resorbed. These results suggested that cells could not infiltrate the non-hybridized specimens because of the absence of macropores.

In contrast to these findings, we observed comparatively unclear border lines between gelatin-hybridized cement specimens and their respective host bones in micro-CT observations, suggesting that the peripheral areas of the specimens were partially resorbed by osteoclastic cells 
provided by surrounding tissues. Histological evaluation revealed, moreover, that the use of chondroitin 6-sulfate in the liquid component could considerably enhance the bioresorption rates of gelatin-hybridized specimens. Most notably nearly $85 \%$ of the specimens were resorbed and replaced by newly formed hypo-calcified and calcified bones within the eight-week implantation period. It was considered that the improved osteoconductivity of the specimens created by the addition of chondroitin 6-sulfate immobilizing growth factors or other cytokines to the specimens, along with the formation of macropores formed by gelatin degradation, could contribute significantly to the bioresorbability enhancement of the gelatinhybridized CPC. Gelatin-hybridized CPCs with chitosan, on the other hand, did not show significant promotion of cement specimen resorption. This may be have been because of their cytotoxicity. ${ }^{18)}$

The most important finding obtained by the present study is that the 10 mass $\%$ gelatin-hybridized chelatesetting IP6-HAp/ $\alpha$-TCP cement containing chondroitin 6-sulfate showed excellent bioresorbability at a relatively early stage of implantation into bone defects of a large animal. Kasuya et al. prepared and implanted a conventional CPC hybridized with 10 mass $\%$ gelatin particles, which were the same as those we used, into trabecular bone defects of the distal femurs of rabbits. ${ }^{17)}$ Their results showed that the areas of the residual CPC at 4, 12, 24 weeks after implantation were $65,54.2 \pm 13.4$, and $31.0 \pm 15.9 \%$, respectively. The bioresorbability of the same gelatin-hybridized conventional CPC was also evaluated by Matsumoto et al., moreover, using a saddle-type bone defect model created in a canine mandible. ${ }^{19)}$ They demonstrated that almost all of the cement specimen was resorbed and replaced by newly formed bone within the implantation period of 24 weeks. Although it is difficult to compare the bioresorbability of the two gelatin-hybridized CPCs due to differences in the experimental designs, gelatin-hybridized chelate-setting IP6-HAp/ $\alpha$-TCP cement is expected to have superior bioresorbability considering the results obtained by the present study.

The two gelatin-hybridized CPCs differed in not only the composition of the powder phase, but also in that of the liquid phase. The concentration of chondroitin sulfate in the gelatin-hybridized chelate-setting IP6-HAp $/ \alpha$-TCP cement, in particular, was higher than that of the gelatinhybridized conventional CPC of 5 mass \%. The increase in the concentration of chondroitin sulfate would therefore enhance the osteoconductivity of the CPC to allow an early appearance of mononuclear cells and osteoclasts around the cement specimen, ${ }^{5)}$ which might result in acceleration of the cement resorption. According to a previous report by $\mathrm{Li}$ et al. that catalytic activity of cathepsin $\mathrm{K}$, the predominant collagenase in osteoclasts, requires the formation of complexes with glycosaminoglycans such as chondroitin sulfate, ${ }^{20)}$ moreover, the gelatin-hybridized chelate-setting IP6-HAp $/ \alpha$-TCP cement was assumed to activate cathepsin $\mathrm{K}$ more effectively to facilitate gelatin degradation.

\section{Conclusions}

Our results suggest that the 10 mass $\%$ gelatinhybridized chelate-setting IP6-HAp/ $\alpha$-TCP cement fabricated with a liquid component, including 15 mass \% of chondroitin sulfate, was a beneficial CPC for non-loadbearing applications, with sufficient bioresorbability and material properties. However, the resorption mechanism of the cement specimens and their replacement by newlyformed bones have not yet been completely elucidated. Whether or not the newly-formed bones would have mechanical strength equivalent to that of the host bones also remains unknown. Further experiments are required to examine these issues.

\section{References}

1) X. Wang, L. Chen, H. Xiang and J. Ye, J. Biomed. Mater. Res. B, 81, 410-418 (2007).

2) M. Takeuchi, Y. Miyamoto, K. Ishikawa, T. Toh, T. Yuasa, M. Nagayama and K. Suzuki, Biomaterials, 19, 2057-2063 (1998).

3) K. Kiminami, K. Matsuoka, T. Konishi, M. Mizumoto, M. Honda, H. Arimura and M. Aizawa, Phosphorus Res. Bull., 33, 7-13 (2017).

4) K. Kiminami, T. Konishi, M. Mizumoto, M. Honda, H. Arimura and M. Aizawa, Materials, 10, 941 (2017).

5) W. Schneider, A. Reinstorf, M. Ruhnow, S. Rehberg, J. Heineck, I. Hinterseher, A. Biewener, H. Zwipp and S. Rammelt, J. Biomed. Mater. Res. A, 85, 638-645 (2008).

6) H. Matsunari, M. Onodera, N. Tada, H. Mochizuki, S. Karasawa, E. Haruyama, N. Nakayama, H. Saito, S. Ueno, M. Kurome, A. Miyawaki and H. Nagashima, Cloning Stem Cells, 10, 313-324 (2008).

7) D. W. Hutmacher, J. T. Schantz, C. X. F. Lam, K. C. Tan and T. C. Lim, J. Tissue Eng. Regen. M., 1, 245-260 (2007).

8) W. J. E. M. Habraken, H. B. Liao, Z. Zhang, J. G. C. Wolke, D. W. Grijpma, A. G. Mikos, J. Feijen and J. A. Jansen, Acta Biomater., 6, 2200-2211 (2010).

9) W. J. E. M. Habraken, J. G. C. Wolke, A. G. Mikos and J. A. Jansen, J. Biomat. Sci.-Polym. E., 17, 1057-1074 (2006).

10) P. Q. Ruhé, E. L. Hedberg-Dirk, N. T. Padron, P. H. M. Spauwen, J. A. Jansen and A. G. Mikos, J. Tissue Eng., 12, 789-800 (2006).

11) Q. Lian, D. C. Li, J. K. He and Z. Wang, P. I. Mech. Eng. H, 222, 347-353 (2008).

12) C. Muzzarelli and R. A. A. Muzzarelli, J. Inorg Biochem., 92, 89-94 (2002).

13) W. J. E. M. Habraken, J. G. C. Wolke, A. G. Mikos and J. A. Jansen, J. Biomed. Mater. Res. B, 91, 555-561 (2009).

14) A. Bigi, S. Panzavolta, L. Sturba, P. Torricelli, M. Fini and R. Giardino, J. Biomed. Mater. Res. A, 78, 739-745 (2006).

15) R. A. Perez, S. D. Valle, G. Altankov and M. P. Ginebra, J. Biomed. Mater. Res. B, 97, 156-166 (2011).

16) W. J. E. M. Habraken, L. T. Jonge, J. G. C. Wolke, L. Yubao, A. G. Mikos and J. A. Jansen, J. Biomed. Mater. Res. A, 87, 643-655 (2008). 
17) A. Kasuya, S. Sobajima and M. Kinoshita, J. Orthop. Res., 30, 1103-1111 (2012).

18) M. Huang, E. Khor and L. Y. Lim, Pharm. Res., 21, 344-353 (2004).

19) G. Matsumoto, Y. Sugita, K. Kubo, W. Yoshida, Y.
Ikada, S. Sobajima, M. Neo, H. Maeda and Y. Kinoshita, J. Biomater. Appl., 28, 1316-1324 (2013).

20) Z. Li, Y. Yasuda, W. Li, M. Bogyo, N. Katz, R. E. Gordon, G. B. Fields and D. Brömme, J. Biol. Chem., 279, 5470-5479 (2004). 